\title{
Are Space-Filling Curves Efficient Small Antennas?
}

\author{
José M. González-Arbesú, Sebastián Blanch, and Jordi Romeu, Member, IEEE
}

\begin{abstract}
The performance of space-filling curves used as small antennas is evaluated in terms of quality factor and radiation efficiency. The influence of their topology is also considered. Although the potential use of these curves for antenna miniaturization their behavior is not exceptional when compared with other intuitivelygenerated antennas.
\end{abstract}

Index Terms-Fractals, monopole antennas, small antennas, wire antennas.

\section{INTRODUCTION:}

\section{SMALL ANTENNAS AND FRACTALS}

A $\mathrm{N}$ antenna is said to be small when its size is much smaller than its operating wavelength. In fact, when it can be enclosed into a radiansphere [1] of radius $a$ (being $a=\lambda / 2 \pi$ ). Small antennas are constrained in their behavior by a fundamental limit stated by Chu and reexamined by McLean [2], [3]. In the end, it is the electrical size $k a$ (being $k$ the wavenumber at the operating wavelength in free space) of the antenna what limits the quality factor of a small antenna.

The quality factor is related with the impedance bandwidth of the antenna. It is supposed that the bandwidth of an antenna could be improved when the antenna efficiently uses the available volume of the radiansphere that surrounds it [4], [5]. In this sense, the properties of fractal geometries were expected to help when trying to design useful antennas that might improve some features of common Euclidean ones [6]. Among them, the fact that fractals have a Haussdorf-Besicovitch dimension that could be fractionary and greater than their topological dimension. Mandelbrot [7] called this dimension fractal dimension.

Recently, a lot of effort is concentrated on demonstrating how fractals of higher fractal dimension are more efficient at lowering resonant frequencies than any other geometry [8] and, conversely, that fractals are not more effective than other geometries with the same wire diameter, total wire length, and occupied area [9], [10].

In our opinion, attention must concentrate on the ability of fractal geometries to efficiently design antennas. This efficiency has to be evaluated in terms of radiation efficiency $(\eta)$ and lossless quality factor $(Q)$ versus the electrical size $k a$ of the antenna, the parameter that according to the fundamental limit defines the performance of the antenna. A first approach to this

Manuscript received January 31, 2003; revised April 14, 2003. This work was supported in part by the Spanish Government through Grant TIC 20012364-C03-01, the Ministerio de Ciencia y Tecnología through the Ramón y Cajal Program (2001), the EC through Project IST 2001-33055, and the Departament d'Universitats, Recerca i Societat de la Informació (DURSI) de la Generalitat de Catalunya, Catalunya, Spain.

The authors are with the Electromagnetics and Photonics Engineering Group, Polytechnic University of Catalonia, Barcelona 08034, Spain, and also the Centre the Tecnologia de Telecomunicacions de Catalunya (CTTC), Catalunya, Spain (e-mail: romeu@tsc.upc.es).

Digital Object Identifier 10.1109/LAWP.2003.816638

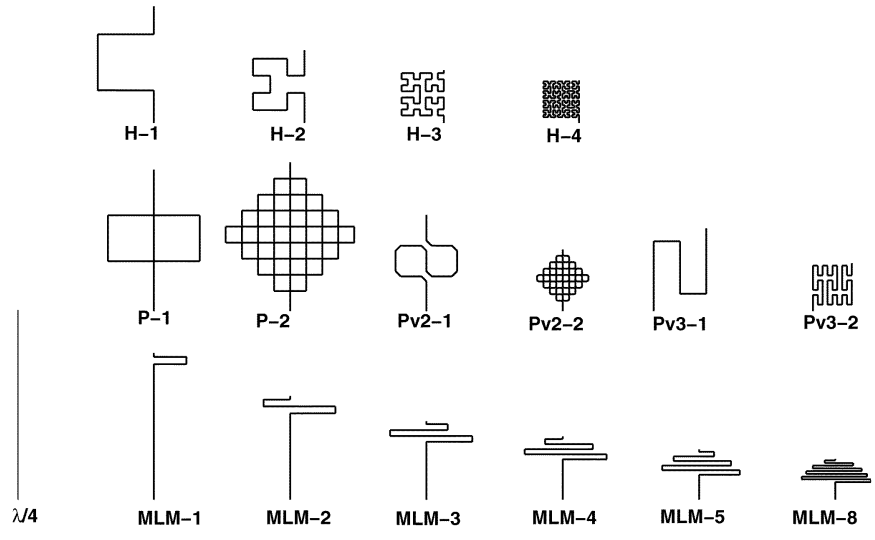

Fig. 1. Bi-dimensional space-filling designs with fractal dimension 2 (for the limit fractal) and Euclidean designs. First row: Hilbert monopoles. Second row: Peano monopoles, Peano variant 2 monopoles, and Peano variant 3 monopoles. Third row: standard $\lambda / 4$ monopole and meander line loaded monopoles.

criterium was presented at [10]. A deeper study assessing how far are fractal antennas at resonance from the fundamental limit and the independence of these results from fractal dimension are presented in this research through the use of quality factor and radiation efficiency maps versus electrical size to show trends of certain fractal families compared against nonfractal designs.

\section{SPACE-Filling CuRves AS Monopoles}

Space-filling fractal curves are potential candidates to build miniature antennas thanks to their capability of compressing large wires into small areas. In [6], a Koch curve was analyzed in a monopole configuration and presented as a first example of fractal curve with improved characteristics. This first experience was extended to fractal dipole configurations using bi-dimensional and three dimensional fractal trees [11]. Simulated configurations agreed with expectations when analyzing the computed $Q$ values for a frequency range where external compensation of the reactive part of the input impedance of the antennas was needed. Nevertheless, for self-resonant prefractal structures (when no external loads are needed to cancel the reactive impedance of the antenna) no practical evidence of their behavior in terms of $Q$ has been reported until now.

The importance of space-filling geometries as optimal or efficient curves for small antenna design is assessed in this work using bi-dimensional wire monopoles. Bi-dimensional geometries are preferred thanks to its easy fabrication procedures with conventional printed circuits manufacturing techniques. Also, monopole configurations are suitable to quick measurement routines because they do not require baluns.

Several plane-filling designs used to build monopole antennas are shown in the first two rows of Fig. 1. All of them are constructed using the iterated function systems (IFS) algorithm 
TABLE I

CHARACTERISTICS OF THE SimUlated MONOPOLES

\begin{tabular}{cccccccc}
\hline Antenna & $\begin{array}{c}\text { Antenna } \\
\text { Height } \\
(\mathbf{m m})\end{array}$ & $\begin{array}{c}\text { Electric Size } \\
\text { at Resonance }\end{array}$ & $\begin{array}{c}\text { Total Wire } \\
\text { Length }(\mathbf{m})\end{array}$ & $\begin{array}{c}\text { Resonant } \\
\text { Frequency } \\
\text { (MHz) }\end{array}$ & $\begin{array}{c}\text { Resonant } \\
\text { Resistance } \\
\text { (Ohms) }\end{array}$ & $\begin{array}{c}\text { Radiation } \\
\text { Resistance } \\
\text { (Ohms) }\end{array}$ & $\begin{array}{c}\text { Quality } \\
\text { Factor }\end{array}$ \\
\hline$\lambda /$ / Monopole & 90,60 & 1,506 & 0,091 & 793,1 & 36,3 & 35,8 & 7,8 \\
Hilbert-1 & 53,50 & 0,8838 & 0,105 & 788,2 & 13,0 & 12,5 & 21,2 \\
Hilbert-2 & 32,77 & 0,5503 & 0,125 & 786,4 & 5,4 & 4,7 & 54,6 \\
Hilbert-3 & 23,30 & 0,4037 & 0,172 & 784,9 & 3,5 & 2,5 & 112,8 \\
Hilbert-4 & 18,50 & 0,3277 & 0,266 & 788,6 & 3,5 & 1,7 & 197,2 \\
Peano-1 & 65,60 & 1,0647 & 0,193 & 774,4 & 14,3 & 14,0 & 11,2 \\
Peano-2 & 69,00 & 1,0711 & 0,605 & 740,7 & 13,9 & 13,8 & 7,4 \\
Peano v2-1 & 44,00 & 0,7274 & 0,117 & 788,8 & 8,3 & 7,7 & 36,9 \\
Peano v2-2 & 27,50 & 0,4555 & 0,209 & 790,3 & 4,8 & 3,3 & 105,3 \\
Peano v3-1 & 37,70 & 0,6262 & 0,109 & 789,6 & 5,3 & 4,7 & 54,6 \\
Peano v3-2 & 21,14 & 0,3714 & 0,174 & 791,8 & 2,5 & 1,4 & 209,7 \\
MLM-1 & 66,80 & 1,1149 & 0,097 & 796,4 & 41,1 & 40,4 & 9,7 \\
MLM-2 & 44,00 & 0,7325 & 0,105 & 794,3 & 17,7 & 17,0 & 17,5 \\
MLM-3 & 32,80 & 0,5439 & 0,119 & 791,2 & 9,6 & 8,9 & 31,3 \\
MLM-4 & 28,60 & 0,4754 & 0,146 & 793,1 & 7,0 & 6,2 & 45,0 \\
MLM-5 & 20,20 & 0,3337 & 0,139 & 788,2 & 3,5 & 2,7 & 89,3 \\
MLM-8 & 12,50 & 0,2033 & 0,141 & 775,9 & 2,0 & 1,1 & 160,5 \\
\hline
\end{tabular}

[12], and all of them generate fractal curves with fractal dimension 2 in the limit. If fractal dimension alone would play a role in the behavior of the antennas we should expect the same performance for all of them. But we cannot work with fractals, we always work with truncated versions called prefractals.

The analyzed monopoles are a Hilbert curve $(H)$ and three variants of a Peano curve. The Hilbert curve and the third variant of the Peano curve (Pv3) are generated using a networked IFS of four and nine transformations, respectively. Another Peano curve $(P)$ is generated using an IFS with nine transformations and with crossings among parts of the curve. Though being an space-filling fractal curve it is not suitable for designing a miniature antenna due to its closed loop structure. To avoid the loops and achieving higher miniaturization ratios the generating procedure is slightly altered and Peano variant 2 curve $(P v 2)$ is obtained although being not strictly a prefractal.

\section{Simulations AND Measurements}

The curves described in Section II have been used to design several wire monopoles. These monopoles are simulated with copper wire of $0.12-\mathrm{mm}$ radius. Simulations include an small 2-mm length segment that accounts for the feeding point where a connector should be welded in a real antenna. Although the lossless models are matched at $800 \mathrm{MHz}$, the antennas are characterized in the range of 500-1500 MHz. Characteristics of simulated monopoles are summarized in Table I. Simulations are carried out using the numerical electromagnetics code (NEC) [13], a method of moments code suitable to analyze wire structures. Each antenna was divided into segments smaller than $0.01 \lambda$ and larger than 2.5 times the wire radius. Extremely short segments smaller than $0.001 \lambda$ are avoided. Prefractals are simulated until iteration 4 for the Hilbert monopole and until iteration 2 for the Peano, Peano variant 2, and Peano variant 3 monopoles. Whereas radiation efficiency calculations were used according

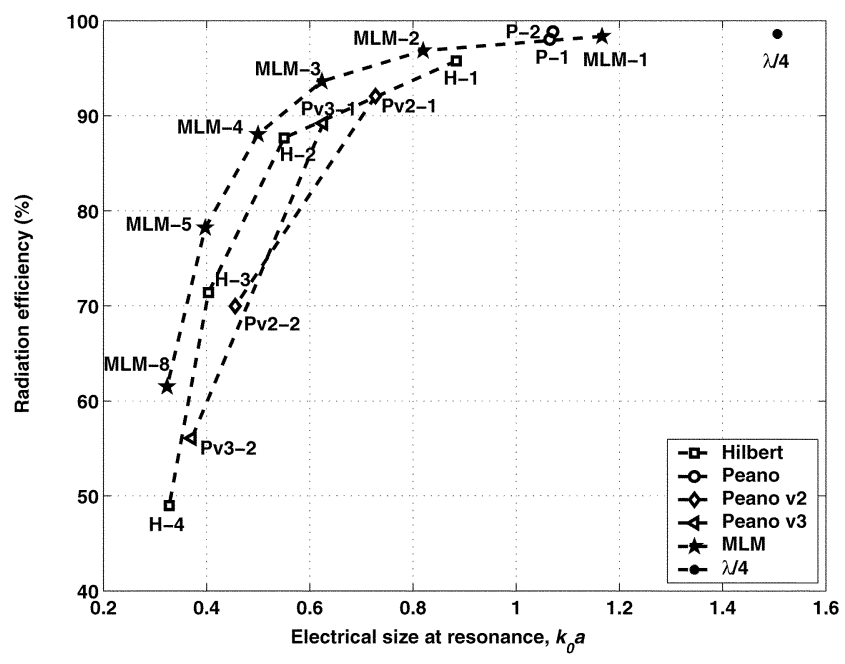

Fig. 2. Computed radiation efficiency at resonance versus electrical size of the antenna. Dashed lines join families of prefractals.

to its definition, the quality factor value was computed with the same definition of Chu [2].

Computed $\eta$ and $Q$ at resonance versus electrical size $\left(k_{0} a\right)$ are shown in Figs. 2 and 3. Dashed lines join prefractals by families. Quality factors are computed for the lossless models of the structures to be compared with the fundamental limit for lineal polarization [3]. As expected, open wire monopoles reduce their radiation efficiency and increase their quality factor with each iteration, while reducing the electrical size of the structure. The increasing wire length with iteration reduces efficiency and, at the same time, the closeness among wire segments cause more intense couplings increasing the quality factor of the structure.

However, the Peano monopole is a closed loop structure and its behavior is quite different. Negligible variations on its electrical size at resonance $\left(k_{0} a\right)$, radiation efficiency and quality factor are noticed while increasing the iteration of the 


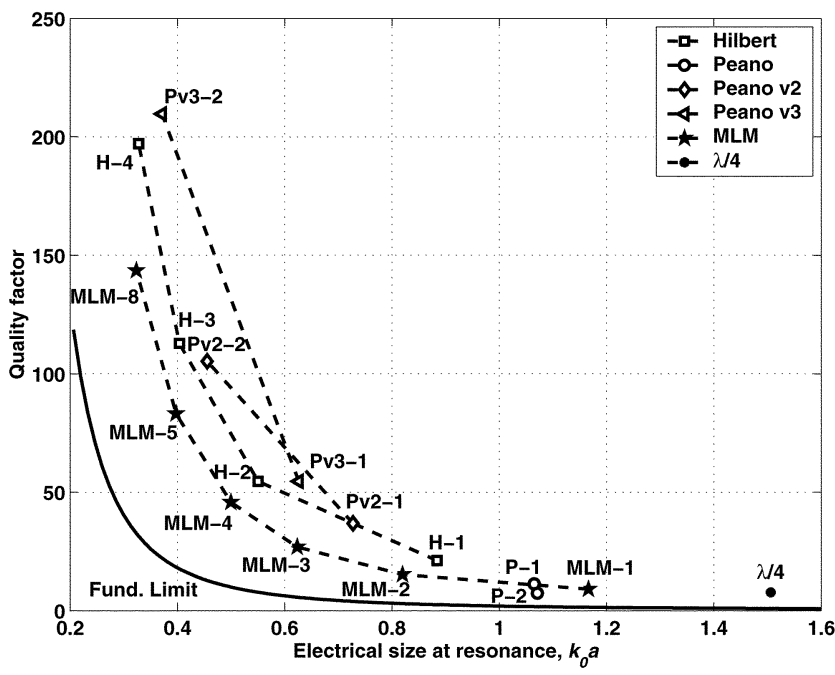

Fig. 3. Computed quality factor at resonance versus electrical size of the antenna. Dashed lines join families of prefractals.

prefractal. It is supposed that with the increasing iteration the Peano monopole tends to behave as a rhombic monopole.

Figs. 2 and 3 reveal that simulations of intuitively generated monopoles loaded with meander lines (shown at the third row of Fig. 1) achieve the same reductions in size with better performances in $\eta$ and $Q$ than prefractals.

The prefractal and the nonprefractal monopoles have been manufactured (Fig. 4) with $0.35-\mathrm{mm}$ strips (25 mm thick) supported on an slim $(0.25 \mathrm{~mm})$ FR4 dielectric substrate. Monopoles are attached to an $80 \mathrm{~cm} \times 80 \mathrm{~cm}$ ground plane and feeded with an SMA connector. They have been measured using the Wheeler cap technique [15] with an small cylindrical radiation shield and making two measurements of the input impedance of the antennas with and without the cap using a network analyzer.

To assess the trend of the simulated Peano monopole an Euclidean rhomb monopole was fabricated and measured. The Peano monopole was fabricated resonant at $1.2 \mathrm{GHz}$ to use the same cap that was employed for the other antennas. The influence of the dielectric substrate and the fabrication of the monopoles with strips and not with wires shifted the resonant frequency of the antennas to lower values than simulated. A $\lambda / 4$ monopole was also fabricated to compare with this well-known antenna the measured frequency shift, the losses in efficiency, and the increase in quality factor due to the presence of the fiberglass substrate and the change of wires by strips. Despite of the substrate and the use of strips, the performance of the monopoles agree with expected simulations as depicted in Figs. 5 and 6: increasing the iteration of the prefractals is useful to reduce the electrical size $\left(k_{0} a\right)$ of the monopoles, but the drastic reduction on radiation efficiency and the increase on quality factor makes these antennas rather unuseful for practical applications.

\section{CONCLUSION}

The behavior of a miniaturized monopole antenna cannot only be evaluated in terms of its size reduction compared with
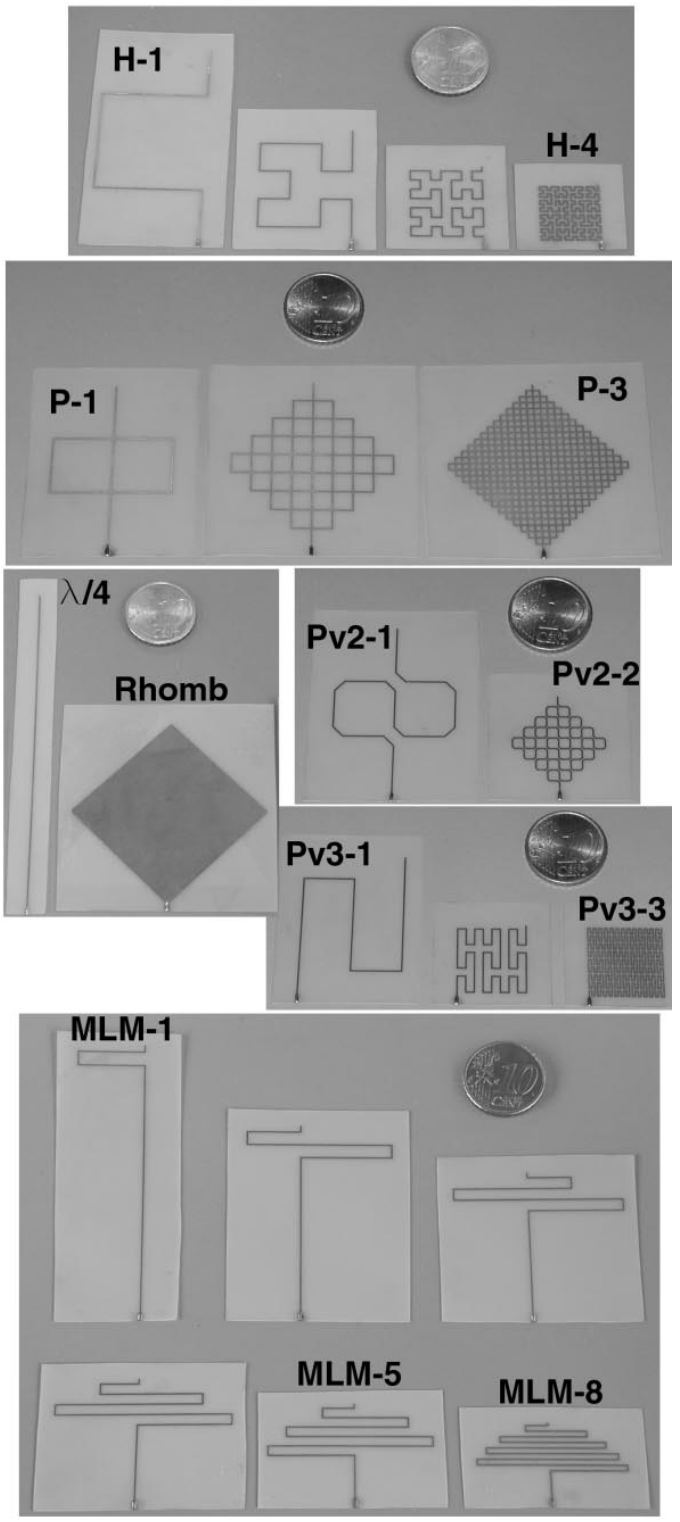

Fig. 4. Fabricated monopoles compared with a 10 eurocent coin. From top to bottom: Hilbert monopoles, Peano monopoles, $\lambda / 4$ monopole and rhomb monopole, Peano variant 2 monopoles, Peano variant 3 monopoles, and meander line loaded monopoles.

an standard $\lambda / 4$ monopole. Of course, this is a very interesting parameter in certain applications, but it is not enough. Another parameters are crucial to define the applicability of the antennas: quality factor and radiation efficiency.

In this sense, space-filling prefractal antennas are not suitable to design efficient miniature antennas. Though suitable for reaching higher miniaturizarion ratios compared with a conventional $\lambda / 4$ monopole, they store a lot of energy in the near fields of the antenna and have higher ohmic losses. Both inconveniences result in high values of quality factors and low values of radiation efficiencies. In addition, and at self-resonance, fractal dimension seems not to play a role in the behavior of the antennas as experiences with prefractals with the same fractal dimension show. These conclusions have been assessed through simulations and measurements on bi-dimensional self-resonant prefractal wire monopoles. 


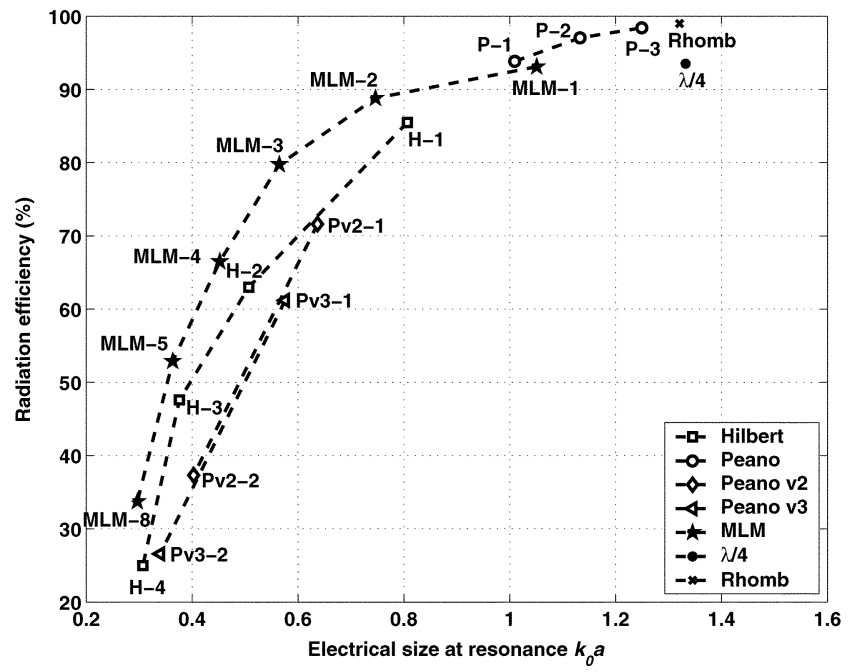

Fig. 5. Measured radiation efficiency at resonance versus the electrical size of the prefractals. Dashed lines join families of prefractals. The performance of a $\lambda / 4$ monopole and a rhomb monopole appear on the graphics.

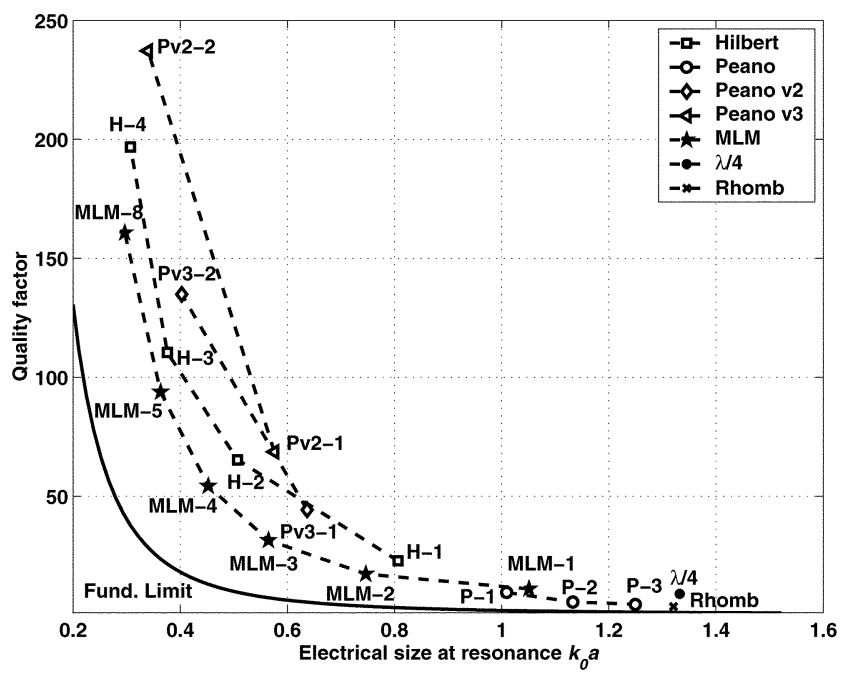

Fig. 6. Measured quality factor at resonance versus the electrical size of the prefractals. Dashed lines join families of prefractals. The performance of a $\lambda / 4$ monopole and a rhomb monopole appear on the graphics.
The experiences from this work also show that other intuitively generated Euclidean configurations perform better than prefractal structures with the same size-reduction ratios $\left(k_{0} a\right.$ values), and even admit more degrees of freedom for the antenna designer.

\section{REFERENCES}

[1] H. A. Wheeler, "Fundamental limitations of small antennas," Proc. IRE, pp. 1479-1484, Dec. 1947.

[2] L. J. Chu, "Physical limitations on omni-directional antennas," J. Appl. Phys., vol. 19, pp. 1163-1175, Dec. 1948.

[3] J. S. McLean, "A re-examination of the fundamental limits on the radiation $Q$ of electrically small antennas," IEEE Trans. Antennas Propagat., vol. 44, pp. 672-676, May 1996.

[4] R. C. Hansen, "Fundamental limitations in antennas," Proc. IEEE, vol. 69, pp. 170-182, Feb. 1981.

[5] C. A. Balanis, Antenna Theory: Analysis and Design. New York: Wiley, 1982, pp. 439-444.

[6] C. Puente-Baliarda, J. Romeu, and A. Cardama, "The koch monopole: A small fractal antenna," IEEE Trans. Antennas Propagat., vol. 48, pp. 1773-1781, Nov. 2000.

[7] B. Mandelbrot, The Fractal Geometry of Nature, W. H. Freeman, Ed. San Francisco, CA: Freeman, 1983.

[8] J. Anguera, C. Puente, E. Martínez, and E. Rozan, "The fractal hilbert monopole: A two-dimensional wire," Microwave Opt. Technology Lett., vol. 36, pp. 102-104, Jan. 2003.

[9] S. Best, "Comments on hilbert curve fractal antenna: A small resonant antenna for VHF/UHF applications," Microwave Opt. Technol. Lett., vol. 35 , pp. 420-421, Dec. 2002.

[10] _ "A comparison of the performance properties of the hilbert curve fractal and meander line monopole antennas," Microwave Opt Technol. Lett., vol. 35, pp. 258-262, Nov. 2002.

[11] J. P. Gianvittorio and Y. Rahmat-Samii, "Fractal antennas: A novel antenna miniaturization tecnique and applications," IEEE Antennas Propagat. Mag., vol. 44, pp. 20-36, Feb. 2002.

[12] H. O. Peitgen, H. Jurgens, and D. Saupe, Chaos and Fractals: New Frontiers of Science. New York: Springer-Verlag, 1992, pp. 231-296.

[13] G. J. Burke and A. J. Poggio, "Numerical Electromagnetics Code (NEC)—Method of Moments," Lawrence Livermore Lab., Livermore, CA, UCID18834, 1981.

[14] E. E. Altshuler, "Electrically small self-resonant wire antennas optimized using a genetic algorithm," IEEE Trans. Antennas Propagat., vol. 50, pp. 297-300, Mar. 2002.

[15] D. M. Pozar and B. Kaufman, "Comparison of three methods for the measurement of printed antenna efficiency," IEEE Trans. Antennas Propagat., vol. 36, pp. 136-139, Jan. 1988. 